\title{
First spectroscopic observations of the substellar companion of the young debris disk star PZ Telescopii ${ }^{\star}$
}

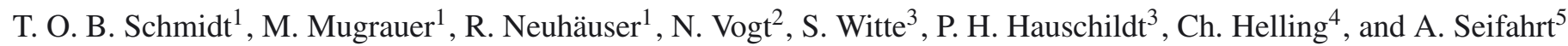

\author{
${ }^{1}$ Astrophysikalisches Institut und Universitäts-Sternwarte, Universität Jena, Schillergäßchen 2-3, 07745 Jena, Germany \\ e-mail: tobi@astro.uni-jena.de \\ 2 Departamento de Física y Astronomía, Universidad de Valparaíso, Avenida Gran Bretaña 1111, Valparaíso, Chile \\ ${ }^{3}$ Hamburger Sternwarte, Gojenbergsweg 112, 21029 Hamburg, Germany \\ ${ }^{4}$ SUPA, School of Physics and Astronomy, University of St. Andrews, North Haugh, St. Andrews KY16 9SS, UK \\ 5 Department of Astronomy and Astrophysics, University of Chicago, 5640 S. Ellis Ave., Chicago IL 60637, USA
}

Received 2 April 2013 / Accepted 26 March 2014

\begin{abstract}
Context. In 2010 a substellar companion to the solar analog pre-main sequence star PZ Tel and member of the approximately $12 \mathrm{Myr}$ old $\beta$ Pic moving group was found by high-contrast direct imaging independently by two teams.

Aims. In order to determine the basic parameters of this companion more precisely and independent of evolutionary models, hence age-independent, we obtained follow-up spectroscopic observations of the primary and companion.

Methods. We used the Spectrograph for INtegral Field Observations in the Near Infrared (SINFONI) at the Very Large Telescope Unit 4/Yepun of ESO's Paranal Observatory in the $H+K$ band and processed the data using the spectral deconvolution technique. The resulting spectrum of the companion was then compared to a grid of DRIFT-PHOENIX synthetic model spectra, a combination of a general-purpose model atmosphere code with a non-equilibrium, stationary cloud and dust model, using a $\chi^{2}$ minimization analysis. Results. We find a best fitting spectral type of G6.5 for PZ Tel A. The extracted spectrum of the substellar companion, at a spatial position compatible with earlier orbit estimates, yields a temperature $T_{\text {eff }}=2500_{-115}^{+138} \mathrm{~K}$, a visual extinction $A_{V}=0.53_{-0.53}^{+0.84} \mathrm{mag}$, a surface gravity of $\log g=3.50_{-0.30}^{+0.51} \mathrm{dex}$, and a metallicity at the edge of the grid of $[\mathrm{M} / \mathrm{H}]=0.30_{-0.30}$ dex.

Conclusions. We derive a luminosity of $\log \left(L_{\mathrm{bol}} / L_{\odot}\right)=-2.66_{-0.08}^{+0.06}$, a radius of $R=2.42_{-0.34}^{+0.28} R_{\text {Jup }}$, and a mass of $M=7.5_{-4.3}^{+16.9} M_{\text {Jup }}$ for the PZ Tel companion, which is consistent with most earlier estimates using photometry alone. Combining our results with evolutionary models, we find a best-fitting mass of about 21 Jupiter masses at an age corresponding to the recently determined lithium depletion age of $7_{-2}^{+4}$ Myr. Hence, the PZ Tel companion is most likely a wide brown dwarf companion in the $12_{-4}^{+8}$ Myr old $\beta$ Pic moving group.
\end{abstract}

Key words. brown dwarfs - stars: pre-main sequence - stars: atmospheres - planetary systems - planets and satellites: formation stars: individual: PZ Tel

\section{Introduction}

In 2010 two groups performing high-contrast direct imaging surveys around young nearby stars (Mugrauer et al. 2010; Biller et al. 2010) independently reported the discovery of a substellar companion around the young solar analog pre-main-sequence star PZ Tel (HD 174429, HIP 92680), a likely member of the $12_{-4}^{+8}$ Myr old $\beta$ Pic moving group (Zuckerman et al. 2001; Torres et al. 2006) having a spectral type of G5-K8 (Spencer Jones \& Jackson 1936; Messina et al. 2010) at an age of $12.8 \pm 2.2 \mathrm{Myr}$ (Tetzlaff et al. 2011).

While not detectable in 2003 because of its proximity to the host star (Masciadri et al. 2005), the substellar companion was first observed on its orbit in 2007 at a projected separation of $0.255 \operatorname{arcsec}(13 \mathrm{au}$ at $51.5 \pm 2.6 \mathrm{pc}$; van Leeuwen 2007), identified by Mugrauer et al. (2010) in archival data of Chauvin et al. (2010) after detection at 0.337 arcsec in 2009 by Mugrauer et al. (2010). Rebull et al. (2008) detected excess emission in the spectral energy distribution of PZ Tel at $70 \mu \mathrm{m}$ with Spitzer/MIPS, indicating the star is surrounded by a low-mass, cold $(\sim 41 \mathrm{~K})$ debris disk. On the basis of further astrometric data, Mugrauer et al. (2012) were able to show that the preliminary determined

^ Based on observations made with ESO Telescopes at the La Silla Paranal Observatory under program ID 087.C-0109(A). orbital parameters of the PZ Tel companion are compatible with this disk being circumbinary (around both components), but not with being circumstellar, and that a semimajor axis of the nearly edge-on orbit not exceeding 25 au is likely.

Both Biller et al. (2010) using NICI at Gemini-South and Mugrauer et al. (2010) using NACO at ESO VLT concluded from photometric data in their discovery papers that the imaged object is likely a brown dwarf companion to the $\beta$ Pic moving group member PZ Tel. Biller et al. (2010) find best-fitting parameters of $2702 \pm 84 \mathrm{~K}$ and $\log \mathrm{g}$ of $4.20 \pm 0.11 \mathrm{dex}$, hence $36 \pm 6 M_{\text {Jup }}$, while Mugrauer et al. (2010) find $2500-2700 \mathrm{~K}$, hence $28_{-4}^{+12} M_{\text {Jup }}$, both using evolutionary models (Chabrier et al. 2000). Neuhäuser \& Schmidt (2012) noted that given this lower mass limit, the PZ Tel companion could even be a planetary mass object, considering the planet definition by Schneider et al. (2011). Recently, Jenkins et al. (2012) derived an age of the system of $24 \pm 3 \mathrm{Myr}$, based on evolutionary models and chromospheric activity of the primary, contrasting with their own age determination of $7_{-2}^{+4} \mathrm{Myr}$ obtained from lithium depletion. This higher age estimate yields a higher effective temperature $2987 \pm 100 \mathrm{~K}$, surface gravity $(\log g) 4.78 \pm 0.10 \mathrm{dex}$, and mass $62 \pm 9 M_{\text {Jup }}$. They also determined the metallicity $[\mathrm{Fe} / \mathrm{H}]$ of the system to be $0.05 \pm 0.20$ dex. 
Table 1. VLT/SINFONI observation log.

\begin{tabular}{llcccccrc}
\hline \hline Object & $\begin{array}{l}\text { JD }-2450000 \\
{[\text { days }]}\end{array}$ & $\begin{array}{c}\text { Date of } \\
\text { observation }\end{array}$ & $\begin{array}{c}\text { DIT } \\
{[\mathrm{s}]}\end{array}$ & NDIT & $\begin{array}{c}\text { Number } \\
\text { of images }\end{array}$ & Airmass & $\begin{array}{r}\text { DIMM }^{a} \\
\text { Seeing }\end{array}$ & $\begin{array}{c}\tau_{0}{ }^{b} \\
{[\mathrm{~ms}]}\end{array}$ \\
\hline PZ Tel & 5795.62984 & 2011 Aug. 22 & 5 & 9 & 16 & 1.17 & 1.35 & 1.2 \\
HIP 94378 & 5795.65222 & 2011 Aug. 22 & 2 & 5 & 1 & 1.11 & $\sim 1.49$ & 1.1 \\
\hline
\end{tabular}

Notes. All observations were done in $H+K$ band and 0.025 mas/spaxel scale (FoV: $0.8 \operatorname{arcsec} \times 0.8$ arcsec). ${ }^{(a)}$ Differential image motion monitor (DIMM) seeing average of all images; ${ }^{(b)}$ coherence time of atmospheric fluctuations.
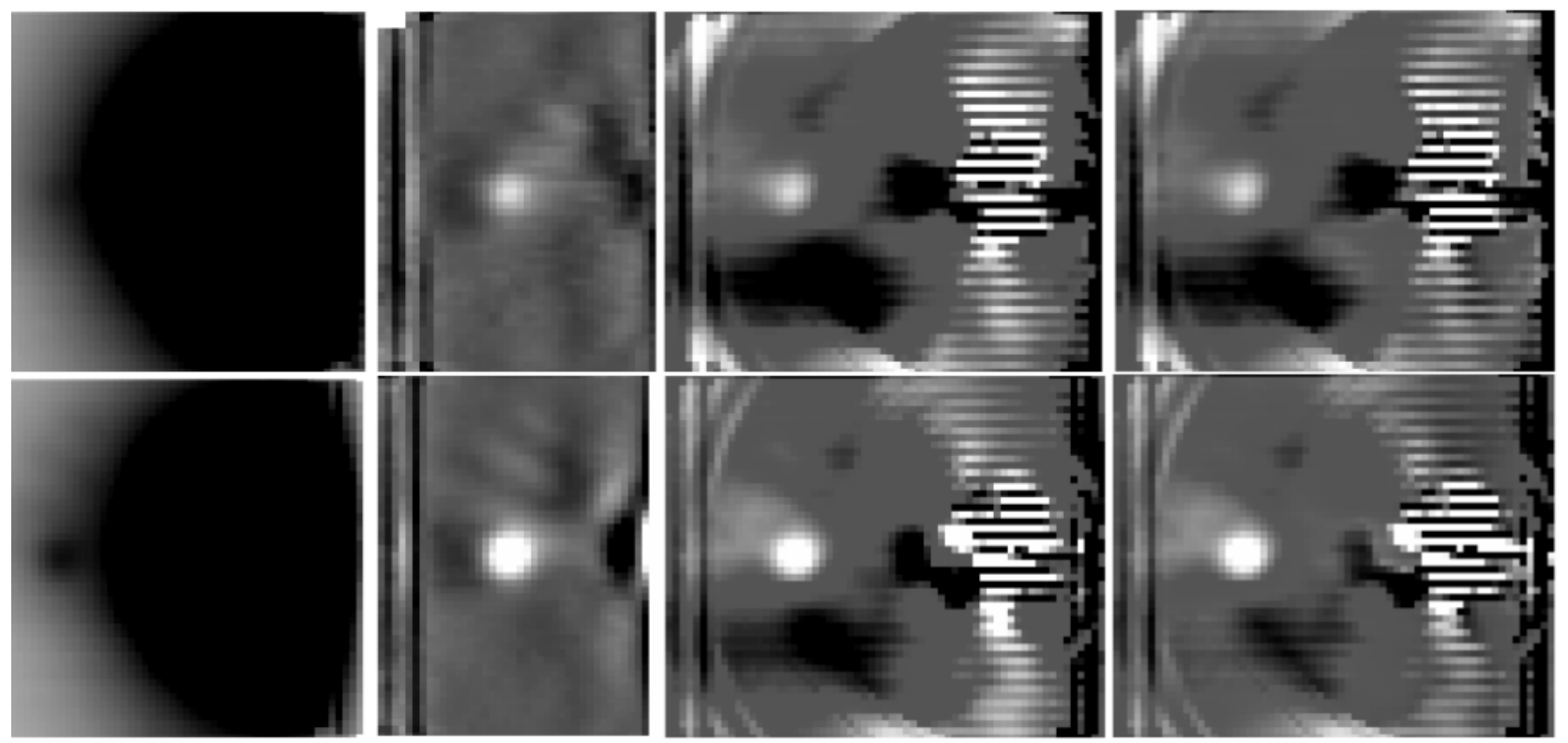

Fig. 1. Averaged data cubes obtained of the PZ Tel companion in $H$ band (upper row) and $K$ band (lower row) at position angle $-30^{\circ}$. From left to right in each row: cube after data reduction, eastern part of cube after two-dimensional polynomial fit of the primary PSF and removal of the primary halo, cube after subtracting the radial symmetric part of the primary PSF, and cube after subtracting the radial symmetric part of the primary PSF and additional spectral deconvolution. For clarity, flux is inverted in the last three images in each row. See text for details.

In order to determine the basic parameters of the PZ Tel companion more precisely and independently of evolutionary models we obtained follow-up spectroscopy. Here we present our results from these observations, targeted to identify the nature of the substellar companion.

\section{Observations and data reduction}

We used the adaptive optics integral-field spectrograph SINFONI, mounted at UT4 of the ESO VLT. The observations of the PZ Tel companion were carried out in the $H+K$ band (resolution 1500) using the best resolving $0.0125 \mathrm{mas} / \mathrm{spaxel} \mathrm{scale} \mathrm{of}$ the instrument with a field of view (FoV) of $0.8^{\prime \prime} \times 0.8^{\prime \prime}$. Further details are summarized in Table 1.

We used the SINFONI data reduction pipeline version 2.0.5 offered by ESO (Jung et al. 2006) with reduction routines developed by the SINFONI consortium (Abuter et al. 2006). After standard reduction, including dark subtraction, flat fielding, distortion correction, and wavelength calibration, all nodding cycles were combined to a final data cube.

We used the Starfinder package of IDL (Diolaiti et al. 2000) and an updated version of an algorithm to remove the halo of PZ Tel by two-dimensional polynomial fit of the primary point spread function (PSF), both described in detail in Seifahrt et al. (2007) and Schmidt et al. (2008). The data quality of July 2011 was not good enough for the data to be used in general; however, for the data of August 2011 we get the result presented as the second image in each row in Fig. 1. The technique was developed for wider companions and the primary being off the FoV, thus an overshooting polynomial removal is present after application here.

Since the primary is in the FoV this additional information can be used for the removal of the primary halo. We use a technique called spectral deconvolution introduced by Sparks \& Ford (2002) for HST data and used for the first time for ground-based data by Thatte et al. (2007). The idea is to remove speckles and other remnants that move with wavelength across the cube and can be distinguished from the non-moving companion by rescaling the cube according to the wavelength of each plane in the cube. Then the companion is at different positions at different wavelength/spectral channels in the new cube, while wavelength dependent speckles and remnants remain at the same positions and can be identified using a median and removed from the original cube (for details of the technique see Thatte et al. 2007). However, it only works if the wavelength coverage and the projected separation of the two components have an appropriate ratio to each other, ensuring that the companion moves enough after rescaling to remove its PSF by median combination of a small fraction of wavelength channels at either end of the data cube. Such a clean collapsed image devoid of flux from the companion can be constructed in our case because the $\epsilon$ value defined in Thatte et al. (2007) easily exceeds unity, being 1.83 for the projected separation of PZ Tel \& its substellar companion (see next section). 
In a first step we have to remove the strongest part of the influence of the halo of the primary, as the halo full width strongly changes with wavelength because of the difference in adaptive optics (AO) performance at different wavelengths. We achieve that by determination and removal of the radial symmetric part of the primary PSF, as can be seen in the third image of each row in Fig. 1. The center determination and reshifting of the PSF was improved even more to the results of Starfinder (Diolaiti et al. 2000) by fitting a second-degree polynomial to the $\mathrm{x}$ shift values of the primary PSF from spectral channel to spectral channel in the cube to suppress any rapid changes on short wavelength differences. This can be neglected for y shift values as the cube was aligned in $y$ according to the position angle of $\sim 60^{\circ}$ of the substellar companion with respect to the primary for the observations.

We then perform the spectral deconvolution technique using a self-written version in IDL. To fully optimize the technique we also remove the PSF of the secondary within an iterative spectral deconvolution implementation in ten iteration steps, as similarly done in Thatte et al. (2007) and arrive at the final images of each row in Fig. 1. As can be seen, not all remnants could be removed. We note that this is expected as the reproducibility of speckles and similar remnants and hence the efficiency of the technique decreases with increasing seeing and decreasing coherence time, both not ideal in our observations (Table 1).

As telluric standard HIP 94378, a B5V star was used for the $H+K$ band. In order to correct for features of this standard star, the Bracket- $\gamma$ line in the $K$ band as well as the $\zeta, \theta, \eta$, and $\iota$ lines of the Bracket series in the $H$ band were removed before usage of the standard. A correction temperature of $15400 \mathrm{~K}$ was assumed for the star, as given for a B5V in Kenyon \& Hartmann (1995). We use the optimal extraction technique by Horne (1986) to improve the quality of the standard, as it was observed at a slightly inferior seeing (Table 1).

We cannot use PSF fitting of the substellar companion as the signal-to-noise ratio $(\mathrm{S} / \mathrm{N})$ is quite low in parts of the $H$ band, hence aperture spectroscopy is our choice. However, we realized that the spectral shape changes with different aperture sizes, a natural outcome of different Strehl ratios achieved at the different wavelength used. Hence, we apply a correction fuction to the extracted spectrum. We extract the spectrum of the primary at the same aperture size as the companion and at the maximum possible aperture size and multiply the companion spectrum by the quotient of these spectra to correct for the differences of AO performance achieved at different wavelengths. The optimal extraction is not used in order not to emphasize artificial deviations.

Without the correction function we find temperatures for the companion about $700 \mathrm{~K}$ lower than our final result. Nevertheless, different aperature sizes still give different results, which can be attributed to different $\mathrm{AO}$ performances achieved for primary and companion and/or remnants in the final cubes not removed by reduction and spectral deconvolution. Because of the proximity of the companion, the AO performance at primary and companion position should be closely comparable. Since we also see strong and spatially close positive remnants in the $K$ band, artificially increasing the flux for increasing aperture sizes, we conclude that these remnants are the dominant source of changes in the spectral shape in accordance with aperture size. Hence, the accuracy of the spectrum should increase with decreasing aperture size, while its precision diminishes.

At the beginning of the $H$ band at $1.45 \mu \mathrm{m}$, the sparrow resolution limit (Sparrow 1916) is at 34.6 mas or 2.8 spaxels, hence we choose an aperture size of 2.5 spaxels. This choice

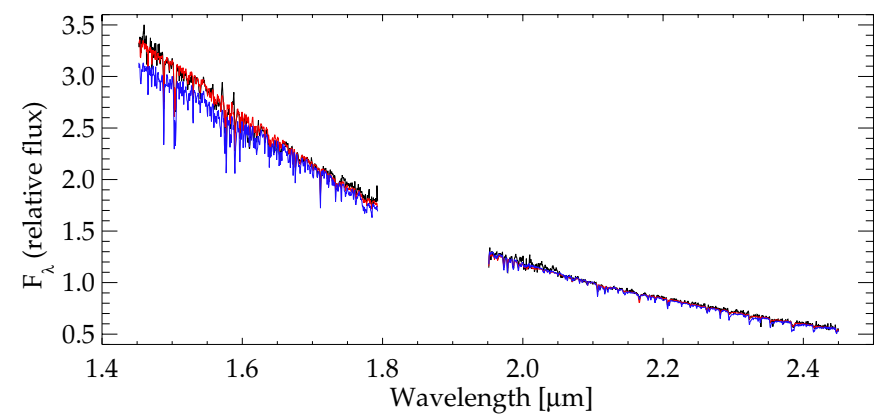

Fig. 2. Standard calibrated spectrum of PZ Tel (black) compared to an IRTF Spectral Library G6.5V comparison spectrum (red/light gray) and a K0V comparison spectrum (blue/gray). See text for details. (Color version online.)

guarantees the best accuracy (without further inclusion of remnants), while a lower value would be below the resolution and comes into conflict with the precision of spatial PSF alignment at different wavelengths.

\section{Results}

\subsection{Astrometry}

An average projected separation of 32 spaxels was found between the components of the PZ Tel system. As no astrometric calibration of SINFONI was observed, we cannot give accurate numbers. However, 32 spaxels correspond to 0.4 arcsec using the nominal spaxel scale of 0.0125 "/spaxel, being closely in agreement with the orbit determination of Mugrauer et al. (2012).

\subsection{Spectroscopy of $P Z T e l$}

As PZ Tel is within the FoV, dominating the obtained flux, we can extract a spectrum of high $\mathrm{S} / \mathrm{N}$. Figure 2 shows the standard calibrated spectrum of PZ Tel (black) in comparison to IRTF (NASA Infrared Telescope Facility) Spectral Library products (Rayner et al. 2009). A best fit is achieved for the G6.5V comparison spectrum (red) from the spectral library, while a K0V (blue), as found by Houk (1993) and commonly given as spectral type (Perryman \& ESA 1997), exhibits a too red spectral continuum. A spectral type of G6.5V corresponds to about $5665 \mathrm{~K}$ (Kenyon \& Hartmann 1995), which agrees well with the temperature estimates of $5623 \mathrm{~K}$ in Allende Prieto \& Lambert (1999) and 5308-6065 K in Bailer-Jones (2011), as well as earlier spectral type determinations as G5 (Spencer Jones \& Jackson 1936). Discrepancies could be attributed to variability, common at the youth of PZ Tel.

\subsection{Spectroscopy of the PZ Tel companion}

As described in the previous section extensive reductions and corrections had to be applied to improve the quality of the extracted spectrum, including standard reduction, rotation subtraction, spectral deconvolution, and application of an AO performance correction function, as well as the best choice of aperture size.

As dust begins to condensate at temperatures $T_{\mathrm{eff}} \approx 2700 \mathrm{~K}$ (Tsuji et al. 1996; Chappelle et al. 2005), we compare the spectrum of the substellar companion to synthetic model spectra taking the influence of dust within a kinetic model description of the formation and evolution of dust in brown 


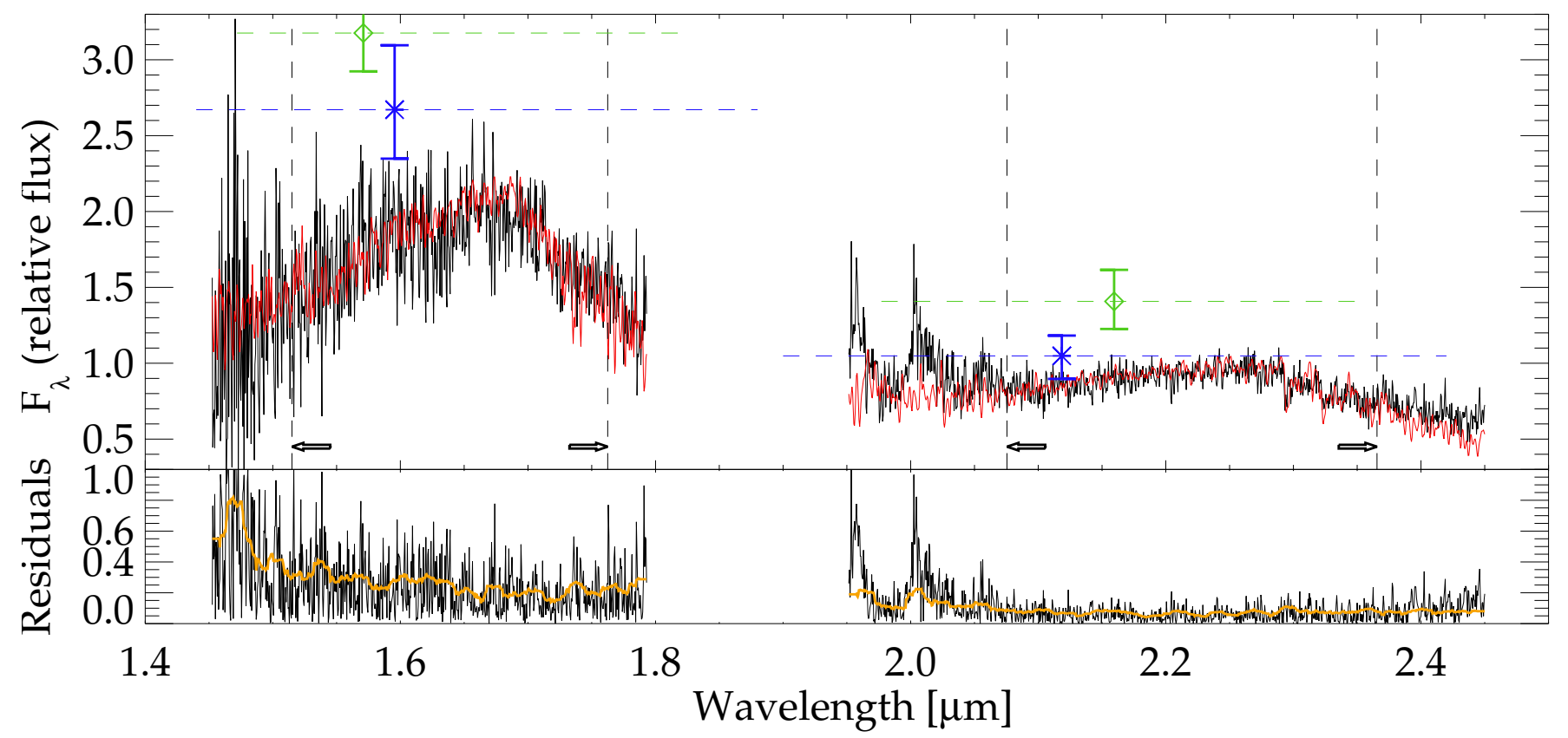

Fig. 3. Top: our SINFONI spectrum of the PZ Tel companion (black) in spectral resolution 1500 compared to the best-fitting DRIFT-PHOENIX synthetic spectrum (red/gray; same spectral resolution) of $T_{\text {eff }}=2500 \mathrm{~K}, \log g=3.5 \mathrm{dex},[\mathrm{M} / \mathrm{H}]=0.3 \mathrm{dex}$, and a visual extinction of $A_{V}=$ $0.53 \mathrm{mag}$. The spectral range used for $\chi^{2}$ minimization is indicated by arrows as well as vertical dashed lines. Magnitude differences with regard to the primary as given in Mugrauer et al. (2010) (blue asterisk) and Biller et al. (2010) (green diamond) were transformed to relative fluxes using the NACO and NICI/NIRI filter transmission curves, respectively. The spectral range of the filters is given as dashed lines, in contrast to negligible spectral error bars. Bottom: absolute value of the residuals of the PZ Tel companion spectrum and the best model fit (black) compared to the determined noise floor (orange/light gray). See text for details. (Color version online.)

dwarfs into account (Helling et al. 2008a). The employed DRIFT-PHOENIX models combine a non-equilibrium, stationary cloud model from Helling et al. (2008b, DRIFT: nucleation, seed formation, growth, evaporation, gravitational settling, convective overshooting/up-mixing, element conservation) with a general-purpose model atmosphere code (Hauschildt \& Baron 1999, PHOENIX: radiative transfer, hydrostatic equilibrium, mixing length theory, chemical equilibrium).

In contrast to Schmidt et al. (2008), we use DRIFT-PHOENIX $\mathrm{v} 1.2$, a complete grid of models with a new equation of state in the range of $T_{\mathrm{eff}}=1000 \ldots 3000 \mathrm{~K}, \log g=3.0 \ldots 5.5$ dex, and $[\mathrm{M} / \mathrm{H}]=-0.6 \ldots 0.3 \mathrm{dex}$ in steps of $100 \mathrm{~K}, 0.5 \mathrm{dex}$, and 0.3 dex, respectively. Moreover, we still need to account for reddening of our spectra by extinction. This correction is important as the effective temperature is highly correlated with the extinction, because both values change the slope of the spectrum in the $H+K$ band.

In order to achieve more precise best-fitting values we linearly interpolated the present DRIFT-PHOENIX v1.2 grid to step sizes of $10 \mathrm{~K}, 0.1 \mathrm{dex}$, and $0.1 \mathrm{dex}$ in temperature, logarithm of surface gravity, and logarithm of metallicity, respectively. We used a $\chi^{2}$ minimization algorithm to find the best-fitting combination of (a) the effective temperature; (b) the surface gravity; (c) the metallicity, and (d) the extinction correction of the measured spectrum, a version similar to the one used in Schmidt et al. (2008), but strongly improved in usability and accuracy, for example by a more precise determination of the noise floor of the data (see Fig. 3).

In the upper panel of Fig. 3 we give the best fit of our spectrum (black) with a reduced $\chi_{\text {red }}^{2}$ of 1.16. The best-fitting DRIFT-PHOENIX model (red) of the companion of PZ Tel has a temperature of $T_{\text {eff }}=2500_{-115}^{+138} \mathrm{~K}$, a visual extinction $A_{V}=$ $0.53_{-0.53}^{+0.84} \mathrm{mag}$, a surface gravity of $\log g=3.50_{-0.30}^{+0.51} \mathrm{dex}$, and a metallicity of $[\mathrm{M} / \mathrm{H}]=0.30_{-0.30}$ dex. An upper limit for the metallicity cannot be given, as the best-fitting value is at the upper edge of the used model grid. To improve on the accuracy we linearly fitted 1,2 , and $3 \sigma$ values to determine the final $1 \sigma$ error. In addition, we give the absolute value of the residuals of the fit (black) in the lower panel of Fig. 3 in comparison to the determined noise floor of the spectrum (orange).

The spectral range used for the $\chi^{2}$ minimization is indicated by arrows and vertical dashed lines in Fig. 3. In this area the average $\mathrm{S} / \mathrm{N}$ is 7.5 for the $H$ band and 12.6 per spaxel for the $K$ band. At a resolution of 1500 in $H+K$ we derive an average spectral scale of about 2.5 spaxels per resolution element. Using this value we determine $\sim 423$ of 1077 data points to be independent spectral measurements. On this basis we can calculate the $\chi^{2}$ values corresponding to 1,2 , and $3 \sigma$ significance of 423-4 (four parameters to fit) degrees of freedom to be 432, 469, and 504, respectively, and after correction for the full number of measured spaxels to be 1100,1194 , and 1283 for 1,2 , and $3 \sigma$, respectively.

These significances are given as contours in Fig. 4 for temperature vs. visual extinction, in Fig. 5 for temperature vs. surface gravity, and in Fig. 6 for temperature vs. metallicity. In addition comparison values from the literature are given as straight lines with $1 \sigma$ error bars as dashed lines, color-coded for different authors or as $1 \sigma$ error ellipses if both values plotted were given. The extinction of the primary PZ Tel by Bailer-Jones (2011) is shown in brown, the temperature, surface gravity, and metallicity (of the primary) as found by Jenkins et al. (2012) in magenta, the temperature as found by Mugrauer et al. (2010) in blue, and the temperature and surface gravity as found by Biller et al. (2010) in green. All comparison values were determined using photometry and evolutionary models. The extinction value of PZ Tel A had to be transformed to visual extinction using the formula given in (Bailer-Jones 2011) as well as to $1 \sigma$ significance. 
T. O. B. Schmidt et al.: Spectroscopic observations of the substellar companion of the young debris disk star PZ Telescopii

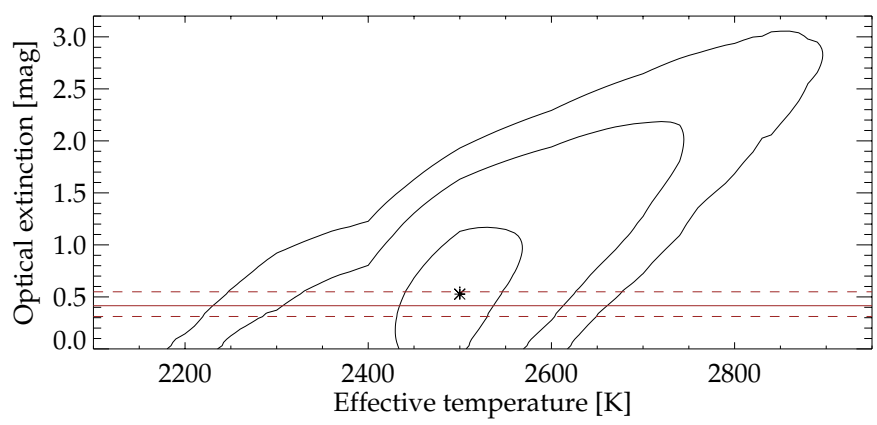

Fig. 4. Result of the $\chi^{2}$ minimization analysis for the PZ Tel companion. Plotted are the best value (asterisk) and the 1,2, and $3 \sigma$ error contours for effective temperature $T_{\text {eff }}$ and optical extinction $A_{V}$, determined from the comparison of our SINFONI spectrum and the DRIFTPHOENIX model grid. In addition, the visual extinction of PZ Tel A is shown (horizontal solid brown line), including its $1 \sigma$ errors (dashed lines) as found by Bailer-Jones (2011).

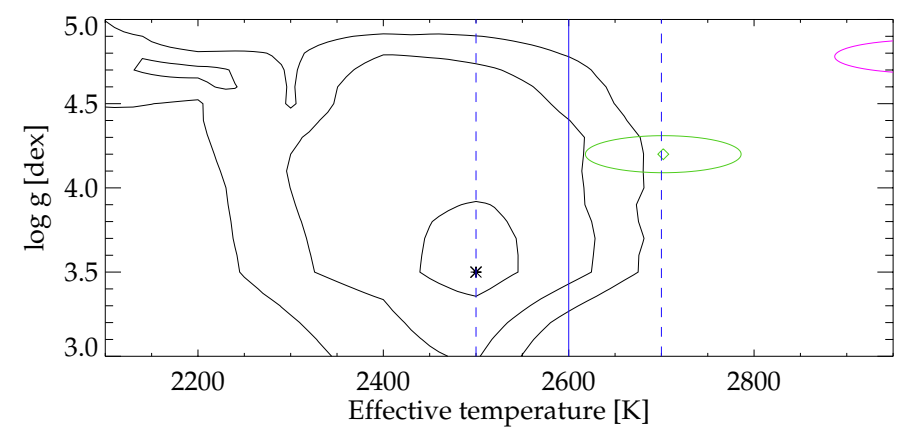

Fig. 5. Result of the $\chi^{2}$ minimization analysis for the PZ Tel companion. Plotted are the best value (asterisk) and the 1,2, and $3 \sigma$ error contours for effective temperature $T_{\text {eff }}$ and logarithm of the surface gravity $\log g$, determined from the comparison of our SINFONI spectrum and the DRIFT-PHOENIX model grid. In addition, the temperature is shown as found by Mugrauer et al. (2010) (vertical solid blue line), including its $1 \sigma$ errors (dashed lines), and surface gravity and temperature as found by (Biller et al. 2010) (green ellipse) and by Jenkins et al. (2012) (magenta part of ellipse).

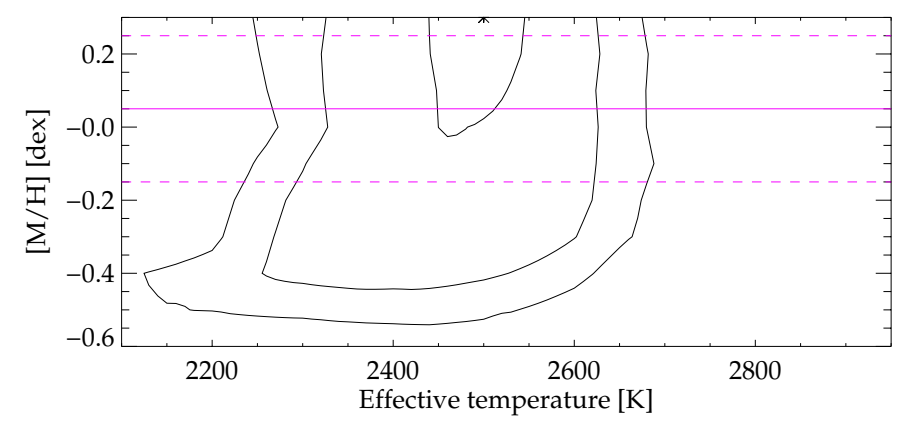

Fig. 6. Result of the $\chi^{2}$ minimization analysis for the PZ Tel companion. Plotted are the best value (asterisk) and the 1,2, and $3 \sigma$ error contours for effective temperature $T_{\text {eff }}$ and metallicity $[\mathrm{M} / \mathrm{H}]$, determined from the comparison of our SINFONI spectrum and the DRIFT-PHOENIX model grid. In addition, the metallicity of PZ Tel A is shown (horizontal solid magenta line), including its $1 \sigma$ errors (dashed lines) as found by Jenkins et al. (2012).

\subsection{Photometry}

In the upper panel of Fig. 3 photometric data points from Biller et al. (2010) (green) and Mugrauer et al. (2010) (blue) are given
Table 2. PZ Tel companion photometry and magnitude differences.

\begin{tabular}{lccc}
\hline \hline Source & $\begin{array}{c}\Delta H \\
{[\mathrm{mag}]}\end{array}$ & $\begin{array}{c}\Delta K \mathrm{~s} \\
{[\mathrm{mag}]}\end{array}$ & $\begin{array}{c}H-K \mathrm{ss} \\
{[\mathrm{mag}]}\end{array}$ \\
\hline Biller $^{a, b}$ & $5.38 \pm 0.09$ & $5.04 \pm 0.15$ & $0.45 \pm 0.18$ \\
This work $^{b}$ & $5.90 \pm 0.16$ & $5.52 \pm 0.09$ & $0.51 \pm 0.19$ \\
Mugrauer $^{c, d}$ & $5.51 \pm 0.09$ & $5.34 \pm 0.06$ & $0.29 \pm 0.12$ \\
This work $^{d}$ & $5.91 \pm 0.20$ & $5.55 \pm 0.11$ & $0.48 \pm 0.23$ \\
\hline maximum sys- & $+\leq 0.7$ & $+\leq 0.8$ & \\
tematic error & $-\leq 0.8$ & $-\leq 0.5$ & \\
\hline
\end{tabular}

Notes. ${ }^{(a)}$ Biller et al. $(2010) ;{ }^{(b)}$ NICI/NIRI filters ; ${ }^{(c)}$ Mugrauer et al. (2010); ${ }^{(d)}$ NACO filters .

for comparison. The magnitude differences given were transformed into flux differences in regard to PZ Tel A by convolving the spectra of PZ Tel and its substellar companion with the filter transmission curves in the $H$ and $K$ s band of NIRI, the same filters as used for the Gemini NICI planet-finding campaign (Liu et al. 2010), and the transmission curves of NACO (Lenzen et al. 2003; Rousset et al. 2003), respectively. As output of the convolution we determine the corresponding fluxes of the previously conducted photometric observations and the effective spectral center of the measurements at which the points are drawn, which differ slightly from the midpoint of the respective filter transmission curves, whose FHWM are shown as horizontal dashed lines in Fig. 3. We note that the full spectral range observed is $1.45-2.45 \mu \mathrm{m}$, used for the convolution, but not shown because of heavy water vapor influence in the case of the challenging observing conditions (Table 1 ). The $H-K$ s color of the companion is constant across all measurements within $0.8 \sigma$ errors.

In Table 2 we present the measured flux differences between PZ Tel and its substellar companion. These differences were measured as described in the previous passage, with the single difference that flux differences were transformed into magnitude differences by the same procedure, i.e. convolving the spectra of PZ Tel and its substellar companion with the filter transmission curves in the $H$ and $K$ s bands of NIRI, the same filters as used for the Gemini NICI planet-finding campaign (Liu et al. 2010), and the transmission curves of NACO (Lenzen et al. 2003; Rousset et al. 2003), respectively. In order to compute an $H-K$ s color of the PZ Tel companion, we assumed $H-K$ s of PZ Tel to be as measured by 2MASS (Cutri et al. 2003; Skrutskie et al. 2006).

In addition we give the maximum possible systematic errors in Table 2, which are present if the strongest positive/negative remnant features in Fig. 1 are superimposed onto the PZ Tel companion spectrum. This amount of remnants is, however, unlikely. On the one hand there is no evident deformation of the substellar companion's PSF, except a brightening towards the northeast (see Fig. 1), which, however, would decrease the magnitude difference between PZ Tel and its substellar companion in the current period (see Table 2). On the other hand, our $H-K$ s color after spectral deconvolution and $\mathrm{AO}$ performance correction among other things, but without the consideration of systematic errors, is only deviant by 0.7 and $0.2 \sigma$ with regard to the results by Mugrauer et al. (2010) and Biller et al. (2010), respectively (Table 2).

\section{Mass determination and conclusions}

Using the photometry of PZ Tel A from the Two Micron All Sky Survey (2MASS) catalog (Cutri et al. 2003; Skrutskie et al. 2006) of $K=6.366 \pm 0.024 \mathrm{mag}$ we can estimate all 
additional parameters of the substellar companion using all results from the spectroscopic analysis described in the previous section. As no absolute photometric calibration is possible with the spectroscopic standard, we make a preliminary estimate of the parameters of the PZ Tel companion assuming negligible photometric variability of both sources, most likely not correct according to the variability indications presented in the previous sections.

We derive a luminosity of $\log \left(L_{\mathrm{bol}} / L_{\odot}\right)=-2.66_{-0.08}^{+0.06}$ for the PZ Tel companion from the extinction corrected apparent brightness $K \mathrm{~s}_{0}=11.86_{-0.10}^{+0.07} \mathrm{mag}$ (from the 2MASS brightness, the magnitude difference (Table 2 ), $A_{V}=0.53_{-0.53}^{+0.84} \mathrm{mag}$, and extinction law by Rieke \& Lebofsky 1985) using a bolometric correction of $B C_{K}=3.1 \pm 0.1 \mathrm{mag}$ from Golimowski et al. (2004, for spectral type M6-L0) at a distance of $51.49_{-2.47}^{+2.74} \mathrm{pc}$ (van Leeuwen 2007). From the luminosity and temperature $T_{\text {eff }}=2500_{-115}^{+138} \mathrm{~K}$, we calculate the radius to be $R=0.25_{-0.04}^{+0.03} R_{\odot}$ or $2.42_{-0.34}^{+0.28} R_{\text {Jup }}$. From radius and surface gravity $\log g=$ $3.50_{-0.30}^{+0.51} \mathrm{dex}$, we find a mass of the PZ Tel companion of $M=$ $0.0071_{-0.0041}^{+0.0161} M_{\odot}$ or $7.5_{-4.3}^{+16.9} M_{\text {Jup }}$.

For the bolometric correction we used the spectral type corresponding to the temperature range found here. Using the $\mathrm{H}_{2} \mathrm{O}$ index defined in Allers et al. (2007) we find a possible spectral range of L1.5-L4 for the substellar companion, using the modified version in Bonnefoy et al. (2014) we find a possible spectral range of M7-L9, roughly consistent with our findings of M6-L0. Bonnefoy et al. (2014) modified the index to avoid noisy regions, definitely necessary in the present case, as the blue part of the $H$ band has the lowest S/N. Our derived surface gravity uncertainties are about equal to the values of 0.5 dex computed by Bonnefoy et al. (2014) for similar objects.

The derived values, shown in Figs. 4-6, agree within $1 \sigma$ with the extinction $A_{V}$ and metallicity $[\mathrm{M} / \mathrm{H}]$ for $\mathrm{PZ}$ Tel A found in Bailer-Jones (2011) and Jenkins et al. (2012), respectively, and with the temperature in Mugrauer et al. (2010). Temperature and surface gravity deviate with regard to literature estimates by 1.2 and $1.3 \sigma$ (Biller et al. 2010) and 2.9 and $2.5 \sigma$ (Jenkins et al. 2012), respectively. Finally, our mass result deviates by $1.2 \sigma\left(28_{-4}^{+12} \mathrm{M}_{\mathrm{Jup}}\right.$, Mugrauer et al. 2010), by $1.6 \sigma\left(36 \pm 6 \mathrm{M}_{\mathrm{Jup}}\right.$, Biller et al. 2010), and by $3.2 \sigma\left(62 \pm 9 \mathrm{M}_{\mathrm{Jup}}\right.$, Jenkins et al. 2012).

Although our mass estimate is independent of evolutionary models, we can use them as comparison to put our results into context and to check which age is indicated by the models for our spectroscopic results. According to Chabrier et al. (2000), DUSTY model best fits are achieved between 5-10 Myr isochrones, fitting our spectral results for temperature, surface gravity, and luminosity within $1 \sigma$ errors. This age range is consistent with the age of the $\beta$ Pic moving group $\left(12_{-4}^{+8} \mathrm{Myr}\right)$, deviant from the age determined by Jenkins et al. (2012) (24 \pm 3 Myr), while very consistent with the lithium depletion age of PZ Tel A of $7_{-2}^{+4}$ Myr by the same authors. Very recently Binks \& Jeffries (2014) combined data of eight low-mass candidates with literature data of $\beta$ Pic moving group members and find a lithium depletion age of $21 \pm 4 \mathrm{Myr}$, which is also inconsistent with the age found here.

Finally, we arrive at a possible mass range of 3.2-24.4 $M_{\mathrm{Jup}}$. According to these estimates the PZ Tel companion is most likely a brown dwarf of about 21 Jupiter masses, as the evolutionary models reject a $\log g=3.5$ dex as only being valid for objects younger than $1 \mathrm{Myr}$ and predict a surface gravity $\log g \sim 3.95$ dex for the given lithium depletion age range, which is within the $1 \sigma$ uncertainty of our results. However, spectra at improved observing conditions and with wider spectral ranges, especially including alkali metal lines for a more precise surface gravity determination, should be able to narrow down the parameters of the PZ Tel companion in the future. Fortunately, such spectra are increasingly easy to acquire because of the large increase in orbital separation, probably persistent for the upcoming years to decades (Mugrauer et al. 2012).

Acknowledgements. We would like to thank the ESO Paranal Team for carrying out the observations as well as the ESO User Support department and all the other very helpful ESO services; as well as the anonymous referee for helpful comments. We would further like to thank Marlies Meyer for helpful discussions of the atmospheric physics and models and Helenka Kinnan for language editing. T.O.B.S. and R.N. would like to acknowledge support from the German National Science Foundation (Deutsche Forschungsgemeinschaft, DFG) in grant NE 515/30-1. N.V. acknowledges support by Comité Mixto ESO-Gobierno de Chile, as well as by the Gemini-CONICYT fund 32090027. Ch.H. highlights financial support of the European Community under the FP7 by an ERC starting grant. This publication makes use of data products from the Two Micron All Sky Survey, which is a joint project of the University of Massachusetts and the Infrared Processing and Analysis Center/California Institute of Technology, funded by the National Aeronautics and Space Administration and the National Science Foundation. This research has made use of the VizieR catalog access tool and the Simbad database, both operated at the CDS, Strasbourg, France. This reasearch makes use of the HIPPARcos Catalogue, the primary result of the HIPPARCos space astrometry mission, undertaken by the European Space Agency. This research has made use of NASA's Astrophysics Data System Bibliographic Services.

\section{References}

Abuter, R., Schreiber, J., Eisenhauer, F., et al. 2006, New Astron. Rev., 50, 398

Allende Prieto, C., \& Lambert, D. L. 1999, A\&A, 352, 555

Allers, K. N., Jaffe, D. T., Luhman, K. L., et al. 2007, ApJ, 657, 511

Bailer-Jones, C. A. L. 2011, MNRAS, 411, 435

Biller, B. A., Liu, M. C., Wahhaj, Z., et al. 2010, ApJ, 720, L82

Binks, A. S., \& Jeffries, R. D. 2014, MNRAS, 438, L11

Bonnefoy, M., Chauvin, G., Lagrange, A.-M., et al. 2014, A\&A, 562, A127

Chabrier, G., Baraffe, I., Allard, F., \& Hauschildt, P. 2000, ApJ, 542, 464

Chappelle, R. J., Pinfield, D. J., Steele, I. A., Dobbie, P. D., \& Magazzù, A. 2005, MNRAS, 361, 1323

Chauvin, G., Lagrange, A.-M., Bonavita, M., et al. 2010, A\&A, 509, A52

Cutri, R. M., Skrutskie, M. F., van Dyk, S., et al. 2003, 2MASS All Sky Catalog of point sources, NASA/IPAC Infrared Science Archive http:// irsa.ipac. caltech.edu/applications/Gator/

Diolaiti, E., Bendinelli, O., Bonaccini, D., et al. 2000, in Proc. SPIE 4007, ed. P. L. Wizinowich, 879

Golimowski, D. A., Leggett, S. K., Marley, M. S., et al. 2004, AJ, 127, 3516

Hauschildt, P. H., \& Baron, E. 1999, J. Comput. Appl. Math., 109, 41

Helling, C., Dehn, M., Woitke, P., \& Hauschildt, P. H. 2008a, ApJ, 675, L105

Helling, C., Woitke, P., \& Thi, W.-F. 2008b, A\&A, 485, 547

Horne, K. 1986, PASP, 98, 609

Houk, N. 1993, VizieR Online Data Catalog: III/51

Jenkins, J. S., Pavlenko, Y. V., Ivanyuk, O., et al. 2012, MNRAS, 420, 3587

Jung, Y., Lundin, L. K., Modigliani, A., Dobrzycka, D., \& Hummel, W. 2006, in Astronomical Data Analysis Software and Systems XV, eds. C. Gabriel, C. Arviset, D. Ponz, \& S. Enrique, ASP Conf. Ser., 351, 295

Kenyon, S. J., \& Hartmann, L. 1995, ApJS, 101, 117

Lenzen, R., Hartung, M., Brandner, W., et al. 2003, in Instrument Design and Performance for Optical/Infrared Ground-based Telescopes. eds. M. Iye, \& A. F. M. Moorwood, Proc. SPIE, 4841, 944

Liu, M. C., Wahhaj, Z., Biller, B. A., et al. 2010, in Proc. SPIE , 7736, 1K

Masciadri, E., Mundt, R., Henning, T., Alvarez, C., \& Barrado y Navascués, D. 2005, ApJ, 625, 1004

Messina, S., Desidera, S., Turatto, M., Lanzafame, A. C., \& Guinan, E. F. 2010, A\&A, 520, A15

Mugrauer, M., Vogt, N., Neuhäuser, R., \& Schmidt, T. O. B. 2010, A\&A, 523, L1

Mugrauer, M., Röll, T., Ginski, C., et al. 2012, MNRAS, 424, 1714

Neuhäuser, R., \& Schmidt, T. O. B. 2012, Topics in Adaptive Optics, ed. R. K. Tyson (InTech) 
T. O. B. Schmidt et al.: Spectroscopic observations of the substellar companion of the young debris disk star PZ Telescopii

Perryman, M. A. C., \& ESA 1997, The HIPPARCOS and TYCHO catalogues. Astrometric and photometric star catalogues derived from the ESA HIPPARcos Space Astrometry Mission, ESA SP, 1200

Rayner, J. T., Cushing, M. C., \& Vacca, W. D. 2009, ApJS, 185, 289

Rebull, L. M., Stapelfeldt, K. R., Werner, M. W., et al. 2008, ApJ, 681, 1484

Rieke, G. H., \& Lebofsky, M. J. 1985, ApJ, 288, 618

Rousset, G., Lacombe, F., Puget, P., et al. 2003, in Adaptive Optical System Technologies II, eds. P. L. Wizinowich, \& D. Bonaccini, Proc. SPIE, 4839, 140

Schmidt, T. O. B., Neuhäuser, R., Seifahrt, A., et al. 2008, A\&A, 491, 311

Schneider, J., Dedieu, C., Le Sidaner, P., Savalle, R., \& Zolotukhin, I. 2011, A\&A, 532, A79

Seifahrt, A., Neuhäuser, R., \& Hauschildt, P. H. 2007, A\&A, 463, 309
Skrutskie, M. F., Cutri, R. M., Stiening, R., et al. 2006, AJ, 131 1163

Sparks, W. B., \& Ford, H. C. 2002, ApJ, 578, 543

Sparrow, C. M. 1916, ApJ, 44, 76

Spencer Jones, H., \& Jackson, J. 1936, Proper motions of stars in the zone catalogue of 20843 stars 1900 (London: HMSO)

Tetzlaff, N., Neuhäuser, R., \& Hohle, M. M. 2011, MNRAS, 410, 190

Thatte, N., Abuter, R., Tecza, M., et al. 2007, MNRAS, 378, 1229

Torres, C. A. O., Quast, G. R., da Silva, L., et al. 2006, A\&A, 460, 695

Tsuji, T., Ohnaka, K., Aoki, W., \& Nakajima, T. 1996, A\&A, 308, L29

van Leeuwen, F. 2007, A\&A, 474, 653

Zuckerman, B., Song, I., Bessell, M. S., \& Webb, R. A. 2001, ApJ, 562, L87 